\title{
Can patients screen themselves? Pilot study of an audio-guided computer-assisted self-interview (ACASI) approach to screening for substance use in primary care
}

\author{
Jennifer McNeely ${ }^{1 *}$, Brian Gilberti ${ }^{1}$, Rubina Khan ${ }^{2}$, John Rotrosen ${ }^{3}$, Shiela Strauss ${ }^{4}$, Marc Gourevitch ${ }^{5}$ \\ From International Network on Brief Interventions for Alcohol Problems (INEBRIA) Meeting 2011 \\ Boston, MA, USA. 21-23 September 2011
}

Lack of a brief, validated screening and assessment tool to identify problematic drug use is a significant barrier to integrating screening, brief intervention and referral to treatment (SBIRT) services into primary care settings. Because patient self-administered screening is potentially more efficient than the traditional face-to-face approach, we undertook a pilot study examining the feasibility and acceptability of an audio-guided computer-assisted selfinterview (ACASI) to identify substance use. We adapted the World Health Organization's Alcohol, Smoking, and Substance Involvement Screening Test (ASSIST) to ACASI format and administered it on touch-screen tablet computers. English- and Spanish-speaking patients were recruited from a large urban primary care clinic. Participants completed the ACASI ASSIST in the waiting area and received a $\$ 4.50$ transit card. Of 47 eligible patients approached, 35 (74\%) agreed to participate. Participants were $57 \%$ male with a mean age of 49 years (range $28-72$ years, $\mathrm{SD}=11)$. The majority $(54 \%)$ were foreign born, 50\% were Hispanic, and 29\% were African American. Twenty-five subjects completed the ASSIST in English, and 10 in Spanish. Thirty participants $(86 \%)$ screened positive for lifetime use of alcohol, and 18 (51\%) for other drugs (excluding tobacco). Twenty-two (63\%) had used alcohol and/or other drugs in the past three months, and 13 (37\%) had moderate- or high-risk use (6 alcohol; 11 other drugs; 4 both). Mean time to complete the ACASI ASSIST was 5.6 minutes (range, 1.517.2 minutes, $\mathrm{SD}=3.2$ ). Responses were $100 \%$ complete. All but one participant felt comfortable answering these questions on a computer. Most either preferred the computer to an interviewer $(50 \%)$ or had no preference (38\%). These results indicate that computer-assisted substance use screening may be feasible and acceptable among a culturally diverse primary care patient population. Our next step will be to evaluate the validity of the ACASI-administered ASSIST.

\begin{abstract}
Author details
'Department of Medicine, New York University School of Medicine, New York, NY, USA. 'Wagner School of Public Service, New York University, New York, NY, USA. ${ }^{3}$ Department of Psychiatry, New York University School of Medicine, New York, NY, USA. ${ }^{4}$ New York University College of Nursing, New York, NY, USA. ${ }^{5}$ Department of Population Health, New York University School of Medicine, New York, NY, USA.
\end{abstract}

Published: 9 October 2012

doi:10.1186/1940-0640-7-S1-A7

Cite this article as: McNeely et al: Can patients screen themselves? Pilot study of an audio-guided computer-assisted self-interview (ACASI) approach to screening for substance use in primary care. Addiction Science \& Clinical Practice 2012 7(Suppl 1):A7. 